\title{
Role of checkpoint inhibitors immunotherapy in non-muscle invasive bladder cancer: current methods and future perspectives
}

\begin{abstract}
Immuno-therapy involvement in bladder urothelial malignancies is growing very fast. The use of Immuno-therapy with check-point inhibitor has greatly developed since it was first approved as a second-line treatment for cases who had formerly failed platinum-based chemotherapy. There are recognized applications for first-line metastatic illness in platinumineligible or cisplatin-ineligible PD-L1 diagnosed cases, as well as a label for BCGrefractory high-risky non-muscle invasive bladder cancer (NMIBC). It is now being studied in neo-adjuvant and adjuvant muscle invasive bladder cancer (MIBC) clinical trials. This review discusses the clinical trials that led to these FDA agreements, as well as prospective and ongoing trials. Current clinical guidelines support Bacillus Calmette-Guérin (BCG) as the primary treating option for intermediate to high-risk NMIBC. Despite the intra-vesical BCG-instillation, intra-vesical relapse occurs in a considerable number of individuals with intermediate to high risk NMIBC. Furthermore, treating BCG-nonresponsive NMIBC is still difficult. For these individuals with BCG-nonresponsive NMIBC, there are no viable therapy alternatives other than radical cystectomy, which has been shown to have excellent oncological results. In this regard, for the care of BCG-nonresponsive NMIBC, safe and reliable noninvasive or lesser-invasive therapeutic alternatives with adequate oncological results are needed. Regarding the latest introduction of immuno-therapeutic medications, the treatment of progressive or metastatic urothelial cancer has substantially advanced. These developments have sparked a surge in interest in immuno-therapeutic medications for NMIBC, particularly BCG-nonresponsive NMIBC. The goal of this literature review is to provide and debate the most up-to-date information on the function of Immuno-therapy in BCG-nonresponsive NMIBC and the presently accessible treatment options. Furthermore, this page highlights the current research in this topic. We wanted to convey the current state of Immuno-therapy in NMIBC and discuss future directions.
\end{abstract}

Keywords: Immuno-therapy, NMIBC, bladder-tumour, urothelial cancers, intra-vesical, Bacillus Calmette-Guérin vaccine (BCG vaccine)
Volume 9 Issue 3 - 202

\author{
Ayman Agag,' Naufal Naushad, ${ }^{2}$ Asad \\ Manzoor, ${ }^{3}$ Sami A Abbas, ${ }^{4}$ Abdalla Ali Deb, ${ }^{5}$ \\ Hosam Serag 6 \\ 'Specialty Doctor in Urology, Frimley Park Hospital, Camberely, \\ United Kingdom \\ ${ }^{2}$ Senior Clinical Fellow in Urology, Wythenshawe Hospital, \\ Manchester University NHS Foundation Trust, Manchester, \\ United Kingdom \\ ${ }^{3}$ Senior Clinical Fellow, Eastbourne District General Hospital, \\ Eastbourne, United Kingdom \\ ${ }^{4}$ Consultant Urologist, National Institute of Urology and \\ Nephrology, Cairo, Egypt \\ ${ }^{5}$ Consultant Urologist, NHS, United Kingdom \\ ${ }^{6}$ Consultant Urologist, University Hospitals Birmingham, United \\ Kingdom
}

Correspondence: Ayman Agag, Specialty Doctor in Urology, Frimley Park Hospital, Camberely, United Kingdom,

Email ayman.agag@nhs.net

Received: August |I, 202 I | Published: November 16, 202 |
Abbreviations: BCG, bacillus calmette-guérin; MIBC, nonmuscle invasive bladder cancer; MIBC, muscle invasive bladder cancer

\section{Introduction}

NMIBC is a kind of bladder tumours that affects the mucosa and sub-mucosa and accounts for around 75 percent of all bladder malignancies. Even though, the majority of the cases with NMIBC respond well after transurethral bladder tumour resection, up to 80 and 15 percent of cases report relapse and progression, correspondingly, within 5 years. NMIBC has a number of pharmacological treatments for preventing recurrence and progression. Intra-vesical instillation of BCG Immuno-therapy is by far the most successfully proven treatment in decreasing recurrence and progression. ${ }^{1}$

The concept of inducing an antitumour immune response in tumour cases dates back to the late 1800 s. Since then, cancer immunotreatments have progressed dramatically, from the intravesical instillation of BCG to the advance of influenceive monoclonal-antibodies built strategies and, most recently, CAR T-cell Immuno-therapy. ${ }^{2}$

Considering the outstanding clinical results acquired by managing numerous solid malignancies with T-cell check-point blockade treatment, the opportunities for immune check-point inhibitors (ICI) remain to expand with several urologic cancers, particularly bladder cancers, being targeted more recently. ${ }^{3}$
New advances in the area of immuno-oncology medications have proved their beneficial outcomes in progressed and metastatic situations, and there is growing suggestion that Immuno-therapy can be used to treat cases who are more indolent. These developments have sparked a surge in interest in immuno-therapeutic medications for NMIBC, particularly BCG-nonresponsive NMIBC. The primary findings of trials looking at the usage of immuno-therapeutic medications for the BCG-nonresponsive NMIBC were lately disclosed, and they show that Immuno-therapy has potential in treating BCGnonresponsive NMIBC. In this sector, several investigations are now being undertaken, and the outcomes of these studies are awaited. The findings of these researches may change the current treatment tactics for BCG-nonresponsive NMIBC, as well as NMIBC generally. ${ }^{4}$ Serial intra-vesical instillations with BCG are one of the first immunomodulatory techniques and the gold standard for NMIBC treating. ${ }^{5}$

Influenceive BCG-treatment has been revealed to prevent tumour recurrences and progressions, which was likened to its potential of triggering a massive influx of inflammatory cells (Th1-polarized lymphocytes and neutrophils), the production of classical activated resident tissues macro-phages (M1), the creation of Th1 cytokines (IFN, IL-12, and TNF-), and the production of anti-tumour cytokines (IFN, IL-12. ${ }^{6}$ However, approximately $30 \%$ of the cases do not respond to BCG-treatment, and growing indications suggest that the preexisting immunological tumour micro-environment influences BCG responsiveness. 
The modulation of the bladder micro-environment for the management of NMIBC via instilling intra-vesical BCG is a classic situation of this, which has been used by urologists for over 40 years. The potential of BCG treatment to elicit a vast influx of Th1-polarized inflammation cells, the formation of Th1 inflammatory cytokines, and the formation of tumour-targeted Th1-intermediated cytotoxic responding is assumed to be the reason for its effectiveness. Even though BCG Immuno-therapy is now optimum for NMIBC treating, 30 percent of cases do not respond to it. ${ }^{8}$

\section{Background for immuno-therapy in BCG nonresponsive NMIBC}

After Morales and colleagues conducted the 1st intra-vesical BCG clinical experiment in 1976, Lamm and colleagues conducted a prospective arbitrary clinical trial to verify the benefits of BCGinstilling in bladder tumor. Yet approximately 30 percent of NMIBC cases don't react to BCG and develop to MIBC. These findings sparked a surge in attention in novel medicines for treating resistant NMIBC, and the instillations of various chemo-therapeutic agents was investigated as a result. However, it was discovered that no one chemo-therapeutic medication can outperform BCG-instilling in terms of effectiveness. The use of various immuno-therapeutic medications for treating systemic bladder cancer was supported by these historical and clinical research. ${ }^{9}$

To produce immuno-therapeutic medications, it is critical to identify novel immuno-therapeutic targets. T-cell regulatory genes were found utilizing molecular biological techniques in the 1980s, and tumour inhibitions with anticytotoxic T-lymphocyte antigen 4 (CTLA4) anti-bodies was revealed in 1996. Numerous other immunological check-points were revealed in the following years. Anti-CTLA4, anti-programmed cell death 1 (PD-1), and anti-programmed cell death ligand 1 are currently the commonest immunological checkpoints (PDL-1). The finding of these immunological check-points, which govern the immune responding, led to the creation of therapy techniques that could be used to influence T-cell activities and generate clinically related anti-tumour influences. These detections prompted the FDA to certify immunological checkpoint inhibitors as a new type of Immuno-therapy, which were accepted for the first time in the United States by a report in 2011 by the FDA. ${ }^{10}$

The usefulness of Immuno-therapy in curing previously systemically treated MIBC was first explored, and significant results were found. In 2016, atezolizumab, a familiar anti-PDL-1 medication, was permitted as a secondary treatment for MIBC based on these findings. Other ICI have now been licensed as secondary treatment for bladder tumours, counting nivolumab, avelumab, durvalumab, and keytruda. Atezolizumab and keytruda were also recognized as firstline treatments for cisplatin-intolerant bladder-tumour. Following licensure, immuno-therapeutic medications' targets for treating NMIBC were quickly identified. ${ }^{11}$

\section{Intra-vesical immuno-therapy}

\section{Intra-vesical immuno-therapy in blend with BCG or as salvage treating after BCG failures}

KLH fights bladder cancer cells by triggering a systemic immune reaction. The treatment methodology calls for a subcutaneous injection to pre-sensitize the product at the time of TURBT, followed by every week intra-vesical instilling in the 1 st and 2 nd months, and then every month injections regimen for a total of 1 year. This may result in an elevated IgG titer that is choosy for bladder cancer cells. Lammers et al. compared the security and effectiveness of KLH and
Mitomycin-C (MM) in intermediate and high-risk NMIBC with no CIS in a prospective arbitrary phase 3 trials. With regard to tumour recurrence, KLH was much worse than MM (61 percent of cases underneath KLH recurred versus $34 \%$ of the cases in the MM group). But, KLH looked to be superior to MM in terms of advancement, particularly after detailed analysis revealed that such a relationship in favor of KLH may be (log-rank test $\mathrm{P}=.049$; univariate Cox regression HR, 0.38 [95 percent $\mathrm{CI}, 0.14$ to 1.04]; $\mathrm{P}=0.059$ ). Both groups experienced similar side influences and had similar security profiles. KLH was shown to be inferior to BCG when compared to it. ${ }^{12}$ Antigens produced from the mycobacterial cell wall are linked to a nucleic acid oligomer combination in MCNA. Morales et al. conducted a phase II single-arm research on 129 cases after BCG failure to assess the effectiveness and security of MCNA. Most of the cases had high-risk illness, with 91 of them having CIS. Overall disease-free survival (DFS) was 19\% in this study after two years. At 2 years, DFS was 32.2 percent in high-risk cases without CIS. At 1 year, the progression-free survival (PFS) rate was 87.3 percent, and at 2 -yrs, it was $79.8 \%$. MCNA was well tolerated and could be a viable choice for bladder-sparing techniques in the future. ${ }^{13}$

More recent research has focused on blend treatments that may improve $\mathrm{BCG}$ response while lowering the required dosage, resulting in better oncological results and a safer profile with less side events that lead to BCG-treatment cessation. Steinberg et al. looked at 52 highrisky NMIBC cases who had previously failed at minimum one BCG treatment and were given six cycles of quadruple Immuno-therapy (intra-vesical $\mathrm{BCG}+\mathrm{INF}+\mathrm{IL}-2$ plus subcutaneous granulocytemacrophage colony-stimulating factor). Only $6 \%$ of the 52 cases were able to tolerate the complete induction. At 1-yr, 55 percent of cases were recurrence-free, and 53 percent were recurrence-free at 2-yrs, eliminating cystectomy in these individuals. ${ }^{14}$

\section{Mechanisms of resistance to BCG}

BCG refractory, resistant, and relapsing individuals are one of the commonest reasons for urgent cystectomy. The upregulation of programmed death ligand-1 (PD-L1) on the tumour cell is a recognized method for evading the immune response elicited by BCG. PD-L1 binds to PD-1, blocking T-cells from initiating an immune response and destroying the bladder tumour cell. As a result, inhibitors that target this check-point have been one of the most researched topics in bladder cancer Immuno-therapy. Recent research has revealed more about the factors that block an anti-tumour immune response, leading to the development of several medications that try to shift the balance from a pro-tumour to an anti-tumour micro-environment, allowing the immune system to combat the tumour. Immunologicalinhibitory check-points, like PD-1/PD-L1, have recently been discovered to operate as potent mediators for cancer's immune escape stages. Targeting both PD-1 and PD-L1 with an ICI could reawaken cytotoxic T cells to combat tumour cells. Additional mechanism of BCG resistances that has been studied is that BCG can inhibit the expression of TNF-mediated $\mathrm{p} 53$, hence reducing cell TNF-mediated apoptosis. We may be able to overwhelm these resisting mechanisms by combination of BCG-treatments with PD-L1 inhibitors (intravesical or systemic) or TNF-, causing a longer response to BCGinstilling, preventing relapse, overcoming resistances and rendering refractory cases sensitive to BCG. Furthermore, we may be capable to decrease the number of the required BCG dosages, lower the risk of adverse influences, permit for longer maintenance treatment and overcome the BCG shortage problem. Many trials are being done based on these findings to investigate the security of intra-vesical Keytruda (NCT02808143) and to match intra-venous and intra-vesical Keytruda in medium-risk NMIBC cases (NCT03167151). The most 
intriguing study is KEYNOTE 676, a randomized controlled phase III trials comparing keytruda plus BCG with BCG alone in high-risky NMIBC cases who have failed to respond to BCG induction. ${ }^{15}$

\section{Systemic immuno-therapy}

On 8 Jan, 2020, the FDA authorized keytruda for the care of cases with BCG-nonresponsive, high-risk NMIBC with carcinoma in situ (CIS) with or without papillary tumours who are unable or unwilling to undergo surgery. Early findings from the KEYNOTE 057 trial, a phase 2 single-arm investigation testing intra-venous keytruda in high-risk NMIBC with BCG failures, showed a responding rate of $38.8 \%$ in cases with a mean period of 8.1-mths, of whom 85.6 percent had a sturdy responding for 6 -mths and 57 percent had a durable responding for 6-mths and 57 percent had a durable reply for 6 Many trials evaluating systemic ICI alone or in conjunction with BCG as part of a multi-modal method with radiotherapy are being done as a result of these encouraging results. ${ }^{16}$

\section{Immuno-therapy for BCG nonresponsive NMIBC: ongoing trials}

\section{Keytruda (Pembrolizumab)}

A number of clinical trials investigating the use of Immuno-therapy in treating NMIBC are now underway. Several researches are now being conducted to examine the use of keytruda in treating NMIBC. KEY-NOTE-676 is an open-label, phase 3, 2 parallel-arm research comparing the security and anticancer effectiveness of keytruda with BCG in high-risky NMIBC cases vs BCG alone (NCT03711032). The open-label, phase 2 single-arm trial examining the influences of blend treatments with keytruda and gemcitabine in BCG-nonresponsive NMIBC is another ongoing research (NCT04164082). Furthermore, a phase 1 single-arm, open-label research is being performed to evaluate the tolerability and effectiveness of blend therapies of intra-vesical keytruda and BCG in high-risk cases or BCG-refractory NMIBC, as well as the tumour features that associate with greater keytruda efficiency (NCT02808143). There are several ongoing clinical trials investigating the usage of Immuno-therapy in treating NMIBC. Notably, there are several researches that continued to assess the usage of keytruda in treating NMIBC. KEY-NOTE-676 is an openlabel, phase 3, 2 parallel-arm research on the security and anticancer activity of blended treatments with keytruda and BCG in comparison with BCG alone in high-risky NMIBC cases (NCT03711032). One more continuing research is the open-label, phase 2, single-arm trial assessing the influences of blended treatments with keytruda and gemcitabine in BCG-nonresponsive NMIBC (NCT04164082). In addition, a phase 1, single-arm, open-label research is being performed on the tolerability and efficacy of blended treatments with intra-vesical administered keytruda and BCG in cases with high risk or BCG-refractory NMIBC. This work aimed as well to study the tumour features that correlate with greater keytruda efficacy (NCT02808143).nCore-001 is a phase 2, open-label, single-arm examining the influence of blended treatments with the oncolytic adenovirus CG0070, intra-venous keytruda, and the transduction improving agent, N-Dodecyl-beta-Maltoside in cases with BCGnonresponsive NMIBC (NCT04387461). ${ }^{17}$

\section{Atezolizumab}

Atezolizumab, an ICI, is as well the subject of numerous active clinical trials in individuals with NMIBC. A phase 1 open label, unrandomized, dosage de-escalation, single-arm trial is being conducted to investigate dosage restrictive toxicity and recurrencefree survival in BCG-naive, high-risk NMIBC cases who receive one intra-vesical BCG installing every week and 1200-mg intra-venous atezolizumab every 3-wks (NCT04134000). The multi-center ALBAN trial compares BCG with atezolizumab in BCG-naive high-risky NMIBC cases against BCG alone in a phase 3 open-label, randomized, 2 parallel-arm trial (NCT03799835). In high-risky NMIBC cases, a phase Ib/II open-label, unrandomized, parallel assigning research has been done to compare the security, tolerability, pharmacology, and antitumour activities of intra-venous atezolizumab mono-treatment versus blended treatments with BCG (NCT02792192). ${ }^{18}$

\section{Durvalumab}

Durvalumab, a PD-L1 inhibitor, has now been studied in a number of clinical trials for treating NMIBC. DURANCE is a phase Ib/II open-label, multicenter, solo arm trial evaluating the security and effectiveness of the 5-peptide cancer vaccination, S-488210/S-488211, in cases with NMIBC who receive the blended treatments for up to 24 weeks (NCT04106115). The security and effectiveness of every week intra-vesical dosing of up to $1000 \mathrm{mg}$ durvalumab in cases with BCG-refractory NMIBC are being investigated in a phase 2 open-label, single-arm research (NCT03759496). The multicenter POTOMAC trial is a phase 3, open-label, randomised, threeparallel-arm experiment comparing the security and effectiveness of durvalumab and inductions plus maintenance BCG versus standard of care BCG in cases with NMIBC (NCT03528694). The phase 1/2 ADAPT-BLADDER trial is a multiarm, multistage, openlabel, randomized, crossover assignment investigation evaluating the security and effectiveness of durvalumab mono-treatment and durvalumab blended treatments with BCG or radiotherapy in cases with BCG-nonresponsive NMIBC (NCT03317158). In cases with BCG-nonresponsive NMIBC, a phase 1 open-label, single-arm, unrandomized, sequential assigning trial is looking into the security and effectiveness of blended therapy with intra-venous durvalumab and vicinium (NCT03258593). The security and effectiveness of intravenous durvalumab administrations in cases with BCG-nonresponsive carcinomas insitu urothelial bladder-tumour are being studied in a phase-II, open-label, single-arm research (NCT02901548). ${ }^{19}$

\section{Avelumab}

In clinical studies for NMIBC-cases, other ICI, like the PD-L1 inhibitor avelumab, have as well been studied. PREVERT is a phaseII open-label, single-arm research in cases with high-risky, BCGnonresponsive NMIBC. Cases will receive one cycle of $10 \mathrm{mg} / \mathrm{kg}$ IV avelumab 5-day earlier Radiotherapy to the whole bladder, and then each 21-day for a overall of 8-cycle (NCT03950362). The ABC trial is a phase- $1 \mathrm{~b}$, single-arm, open-label research assessing the impacts of avelumab and intra-vesical BCG combined therapy in cases with BCG-nonresponsive NMIBC (NCT03892642) ${ }^{20}$

\section{Nivolumab}

Current clinical trials are also examining into the use of the PD-1 check-point inhibitor nivolumab in the cure of NMIBC. In cases with BCG-nonresponsive NMIBC, Check Mate 9UT is a phase 2 open-label, randomized, analogous assignments research comparing the impacts of nivolumab mono-treatment with a combination of nivolumab and experimentally BMS-986205 given with or with no BCG (NCT03519256). ${ }^{21}$

\section{PF-06801591}

Lastly, a phase-III, open-label, randomized, 3 parallel-arm research has been performed on the blended usage of the experimental PD-1 check-point inhibitors, PF-06801591 and BCG vs. treating with BCG only in high-risky NMIBC cases and no previous BCG treatments (NCT04165317).22 


\section{Immune check-point inhibitors (ICI)}

$\mathrm{BC}$ cells can produce PD-L1, a protein that seems to prevent the immune system from detecting and attacking tumour cells. PD-L1 is detected in the tumour micro-environment of $\mathrm{BC}$ cases in the invasive and meta-static stages. Evidence suggests that PD-L1 interacts to the protein PD-1, which is existing on T-lymphocytes. T-cell infectivity is caused by the interactions of PD-L1 and PD-1. The immune system's ability to detect and eradicate tumours is restored by inhibiting PDL1 molecules (Carneiro et al., 2015). In 31 cases with meta-static UCC, MPDL3280A, a mono-clonal-antibodies targeting PD-L1, was examined. The results were encouraging; aside from a $50 \%$ responding rate, side effects were found to be less severe than chemotherapy and tolerable in cases with renal insufficiency. ${ }^{23}$

Given the difficulties of therapy and the risks of recurrences and progressions in cases with BCG-refractory diseases, the FDA approved keytruda in Jan 2020 for treating BCG-refractory, high-risk NMIBC with CIS with or with no papillary tumours in cases who are either ineligible for or have declined cystectomy. ${ }^{24}$ The clinical trial KEYNOTE-057 (NCT02625961) involved 148 cases for its singlearm trial, with 96-cases having BCG-refractory CIS. Of the 148-cases, $41 \%$ had a 3-mths comprehensive responding (urine cytology, negative cystoscopy, and CTU), with a median responding period of 16.2-mths.56 A following ongoing phase-3 trial, KEYNOTE-676 (NCT03711032) enrolled 96. ${ }^{25}$ The aim is to see if this combination medication is influenceive and safe.

Previously, keytruda was solely used to treat locally progressive or meta-static cancer. It is presently used as a secondary treatment for people who have failed or become intolerant to platinum-based chemo-therapy. This remarkable break-through in the realm of Immuno-therapy may offer for more treatments alternatives for cases that have formerly unsuccessful other mainstream therapies as various trials proceed. ${ }^{26} \mathrm{Up}$ to our best knowledge there are no trials assessing ICI in NMIBC. While PD-L1 was revealed frequently in MIBC and meta-static UCC the inhibitions of PD-L1 in sub-types of BC (i.e., high-risk) can represent a promising treating tool in cases with $\mathrm{BCG}$ failures to investigate.

\section{Interleukin- I 0 antibody}

BC cells can produce PD-L1, a protein that seems to prevent the immune system from detecting and attacking cancer cells. PD-L1 is detected in the tumour micro-environment of $\mathrm{BC}$ cases in the invasive and meta-static stages. Evidence suggests that PD-L1 interacts to the protein PD-1, which is present on T-lymphocytes. T-cell infectivity is caused by the interactions of PD-L1 and PD-1. The immune system's ability to detect and eradicate tumours is restored by inhibiting PD-L1 molecules. ${ }^{27}$ In 31 cases with metastatic UCC, MPDL3280A, a monoclonal-antibodies targeting PD-L1, was examined. The results were encouraging; aside from a 50\% responding rate, side influences were found to be less severe than chemotherapy and tolerable in cases with renal insufficiency. ${ }^{28}$

Given the difficulties of treatments and the dangers of recurrences and progressions in cases with BCG-refractory diseases, the FDA approved keytruda in Jan 2020 for treating BCG-refractory, high-risk NMIBC with CIS with or with no papillary tumours in cases who are either unentitled for or have refused cystectomy. ${ }^{29}$ The clinical trial KEYNOTE-057 (NCT02625961) involved 148-cases for its singlearm trial, with 96-cases with BCG-refractory CIS. Of the 148 cases, 41 $\%$ had a 3-mths full responding (urine cytology, negative cystoscopy, and CTU), with a median responding period of 16.2-mths.56 A following continuing phase-3 trial, KEYNOTE-676 (NCT03711032) involved 96-cases. ${ }^{30}$ The goal is to see if this combination medication is influenceive and safe.

Previously, keytruda was solely used to treat locally progressive or meta-static cancer. It is presently used as a secondary treatment for people who have failed or become intolerant to platinum-based chemo-therapy. This remarkable breakthrough in the realm of Immuno-therapy may offer for more treatments alternatives for cases that have formerly unsuccessful other mainstream therapies as various trials proceed. ${ }^{31}$

To our information there are no trials assessing ICI in NMIBC. While PD-L1 was revealed frequently in MIBC and meta-static UCC the inhibitions of PD-L1 in sub-types of surface BC (i.e., high-risks) can represent a hopeful treating tool in cases with BCG disappointment to investigate.

An experimental investigation has evaluated the use of a monoclonal antibodies against IL-10 (anti-IL10R1) in combination with contemporaneous BCG-instilling in a murine model of BC. The TH1-responding is recognized to influence BCG effectiveness; on the other hand, IL-10 is known to decrease the TH1-responding and, as a result, is linked to BCG disappointment. The authors discovered a $22 \%$ regression rate in the combined arm of the trial, in comparison with a $6 \%$ regression rate in a group receiving solely BCG treatment. Furthermore, no metastases were discovered in the mice. As a result, the mixture of anti-IL-10R1 mono-clonal antibodies with BCG represents a novel prospective treatment strategy in high-risky NMIBC, merging local and systemic anticancer influences. ${ }^{32}$

\section{Vaccine-based therapy}

Vaccine-founded agents were lately assessed in NMIBC cases. These treatment concentrations on vaccine-founded proteins with the capability to guide the immune system towards bladder tumour definite antigens. There are numerous vaccine-built agents, like recMAGE-A3, ALT-803, ALT-801, PANVAC, and HS-410 presently in clinical studies. The usage of "tumour vaccines" is a hopeful new Immuno-therapy method but more clinical studies are essential to translate these novel modalities into clinical routines. ${ }^{33}$

\section{Ongoing investigations and upcoming directions}

The upcoming of Immuno-therapy in bladder cancer is hopeful, encompassing a wide spectrum of diseases states from non-muscle invasive to meta-static diseases. As our science lasts to progress, the use of the unique genetic profiles of specific tumours will cause more targeted therapies that are better tolerated and possibly more cost effective than old chemotherapy. But the function of targeted therapies at this time is still restricted to a marginal few of cases. While the Immuno-therapy job is strongly recognized in nearly all phase of the diseases, present researches regarding the best primary meta-static method is still growing. It is progressively becoming clear that chemotherapy still the best primary treatment. Trials matching immuno-therapeutic agents and outdated models of chemotherapy are vast and varied and already happening. Maintenance Immuno-therapy was revealed to be of advantage afterward responding or stable diseases to platinum-founded chemotherapy while the frank management of blend chemo- with Immuno-therapy mayn't be measured standard of care at this time. The selection of agents and interchangeability from avelumab to keytruda is still a topic of examination since the previous was indeed exanimated in a phase- 3 setting though the latter is more suitable with more prolonged dosing. As new agents last to 
be expressed and tested, involving the usage of antibody medication conjugates with enfortumab vedotin which is a strong new adding to the treating landscape of urothelial cancers, we suppose to see a great deal of asset and research into the ever-expanding field of Immunotherapy. ${ }^{34}$

\section{Conclusion}

The development in the management of progressive bladder cancer in recent years have led to new horizon of treatment and survival and much of these enhanced results are echo of the growth of the usage of Immuno-therapy. While first-line treatment is characteristically anchored with a cisplatin or platinum-founded regimen, Immunotherapy is gradually being utilized in concert with first-line platinumbuilt treatment as well as it is utilized as maintenance treatment for cases treated with platinum-based therapy and as second-line therapy in those who progressed after platinum-based treatment. It is also used in primary settings in those who are not candidates for platinum-based treatment. All over the world, clinical trials discovering the usefulness of Immuno-therapy will continue to form the future of bladder cancer treatment.

\section{Acknowledgments}

None.

\section{References}

1. Cassell A, Yunusa B, Jalloh M, et al. Non-muscle invasive bladdertumour: a review of the current trend in Africa. World journal of oncology. 2019;10(3):123-131.

2. Chang SS, Stephen A, Boorjian, et al. "Diagnosis and treatment of nonmuscle invasive bladder-tumour: AUA/SUO guideline." The Journal of urology. 2016;196(4):1021-1029.

3. Van Den Bulk J, Verdegaal EM, De Miranda NF. Cancer Immuno-therapy: broadening the scope of targetable tumours. Open Biol. 2018;8(6):180037.

4. Annels NE, Simpson GR, Pandha H. Modifying the non-muscle invasive bladder-tumour immune micro-environment for optimal therapeutic responding. Frontiers in oncology. 2020;10:175.

5. Babjuk A, Bohle M, Burger, et al. EAU Guidelines on Non-Muscleinvasive Urothelial Carcinoma of the Bladder: update. Eur Urol. 71(3):447-461

6. Kitamura H, Tsukamoto T. Immuno-therapy for urothelial carcinoma: current status and perspectives. Cancers. 2011;3:3055-3072.

7. Nunez-Nateras R, Castle EP, Protheroe CA, et al. Predicting responding to bacillus Calmette-Guérin (BCG) in cases with carcinoma in situ of the bladder. Urol Oncol. 2014;32:45.e23-30.

8. Herr HW, Morales A. History of bacillus Calmette-Guerin and bladdertumour: an Immuno-therapy success story. J Urol. 2008;179:53-56.

9. Pettenati, Caroline, Molly A, et al. "Mechanisms of BCG Immunotherapy and its outlook for bladder-tumour." Nature reviews Urology. 2018;15(10):615-625.

10. Cadranel, Jacques, Anthony Canellas, et al. Pulmonary complications of immune check-point inhibitors in cases with nonsmall cell lung cancer. European Respiratory Review. 2019;28:153.

11. Daro-Faye M, Kassouf W, Souhami L, et al. Combined radiotherapy and Immuno-therapy in urothelial bladder-tumour: Harnessing the full potential of the anti-tumour immune responding. World Journal of Urology. 2021;39(5):1331-1343.

12. Lilly, Eddy, Khalil El Gharib. "Immuno-therapy and non-muscleinvasive bladder-tumour: an idea from the 19th century. Expert Review of Anticancer Therapy. 2021;1-4.
13. Larsen, Emilie Stavnsbjerg, Ulla Nordström Joensen, et al. "Bacillus Calmette-Guerin Immuno-therapy for bladder-tumour: a review of immunological aspects, clinical influences and BCG infections." Apmis. 2021;128(2):92-103.

14. Hassler, Melanie R., Shahrokh F, et al. Salvage therapeutic strategies for bacillus Calmette-Guerin failure. Current opinion in urology. 2019;29(3):239-246.

15. Seebacher NA,AE Stacy, GM Porter, et al. Clinical development of targeted and immune based anti-cancer treatments. Journal of Experimental \& Clinical Cancer Research. 2019;38(1):1-39.

16. El Gharib, Khalil, Eddy Lilly. Check-point inhibitors in BCGnonresponsive nonmuscle invasive bladder-tumour: can they help spare the bladder?." Immuno-therapy. 2021.

17. Pfail, John L, Andrew B, et al. Immuno-therapy in non-muscle-invasive bladder-tumour: Current status and future directions. World Journal of Urology. 2020;39(5):1319-1329.

18. John L, Pfail, Andrew B. Immuno-therapy in non-muscle-invasive bladder-tumour: Current status and future directions." World Journal of Urology. 2020;1-11.

19. Nykopp, Timo K, Jose Batista da Costa, et al. Current clinical trials in non-muscle invasive bladder-tumour." Current urology reports. 2018;19(12):1-13.

20. Patel S, Daneshmand S, Lotan Y, et al. Effectiveness and security of blue light flexible cystoscopy with hexaminolevulinate in the surveillance of bladder-tumour: a phase iii, comparative, multicenter study. $J$ Urol. 2018;199:1158-1165.

21. Cella, David, Viktor Grünwald. Case-reported outcomes of cases with advanced renal cell carcinoma treated with nivolumab plus ipilimumab versus sunitinib (CheckMate 214): a randomised, phase 3 trial. The Lancet Oncology. 2019;20(2):297-310.

22. Constantinidou, Anastasia, Constantinos Alifieris, et al. Targeting programmed cell death-1 (PD-1) and ligand (PD-L1): a new era in cancer active Immuno-therapy. Pharmacology \& therapeutics. 2019;194:84-106.

23. Newton MR, Askeland EJ, Andresen ED, et al. Anti-interleukin-10R1 monoclonal-antibodies in combination with bacillus Calmette - Guérin is protective against bladder-tumour metastasis in a murine orthotopic tumour model and demonstrates systemic specific anti-tumour immunity. Clin Exp Immunol. 2014;177:261-268.

24. Balar GS, Kulkarni EM, Uchio, et al. Keynote 057: phase II trial of Keytruda (pembro) for cases (pts) with high-risk (HR) nonmuscle invasive bladder-tumour (NMIBC) nonresponsive to bacillus calmetteguérin (BCG). J Clinical Oncology. 2019;37(7_suppl):350.

25. Kamat N, Shore N, Hahn, et al. KEYNOTE-676: phase III study of BCG and keytruda for persistent/recurrent high-risk NMIBC. Future Oncol. 2020;16(10).

26. Suzman S, Agrawal YM, Ning, et al. FDA Approval Summary: atezolizumab or Keytruda for treating Cases with Advanced Urothelial Carcinoma Ineligible for Cisplatin-Containing Chemo-therapy. Oncologist. 2019;24(4):563-569.

27. Carneiro BA, Meeks JJ, Kuzel TM, et al. Emerging therapeutic targets in bladder-tumour. Cancer Treat Rev. 2015;41:170-178.

28. Newton MR, Askeland EJ, Andresen ED, et al. Anti-interleukin-10R1 monoclonal-antibodies in combination with bacillus Calmette - Guérin is protective against bladder-tumour metastasis in a murine orthotopic tumour model and demonstrates systemic specific anti-tumour immunity. Clin Exp Immunol. 2014;177:261-268.

29. Balar GS, Kulkarni EM, Uchio, et al. Keynote 057: phase II trial of Keytruda (pembro) for cases (pts) with high-risk (HR) nonmuscle invasive bladder-tumour (NMIBC) nonresponsive to bacillus calmetteguérin (BCG). J Clinical Oncology. 2019;37(7_suppl):350. 
30. Kamat N, Shore N, Hahn, et al. KEYNOTE-676: phase III study of BCG and keytruda for persistent/recurrent high-risk NMIBC. Future Oncol. 2020;16(10).

31. Suzman S, Agrawal YM, Ning, et al. FDA Approval Summary: atezolizumab or Keytruda for treating Cases with Advanced Urothelial Carcinoma Ineligible for Cisplatin-Containing Chemo-therapy. Oncologist. 2019;24(4):563-569.

32. Scheidhauer K, Seidl C, Morgenstern A, et al. Treatment of bladder-tumour with Bi-213-anti-EGFRMAb - A pilot study. J Nucl Med. 2014;55(Suppl 1):639.
33. Boehm BE, Svatek RS. Novel therapeutic approaches for recurrent nonmuscle invasive bladder-tumour. Urol Clin North Am. 2015;42:15968.

34. Erck J, Aragon-Ching. Maintenance avelumab for meta-static urothelial cancer: a new standard of care. Cancer Biol Ther. 2020;1-2. 